ARTICLE

Received 17 Jun 2013 | Accepted 8 Nov 2013 | Published 27 Nov $2013 \quad$ DOl: 10.1038/ncomms3899

\title{
Pits confined in ultrathin cerium(IV) oxide for studying catalytic centers in carbon monoxide oxidation
}

Yongfu Sun ${ }^{1, \star}$, Qinghua Liu ${ }^{2, \star}$, Shan Gao ${ }^{1}$, Hao Cheng ${ }^{2}$, Fengcai Lei ${ }^{1}$, Zhihu Sun², Yong Jiang ${ }^{2}$, Haibin $\mathrm{Su}^{3}$, Shiqiang $\mathrm{Wei}^{2} \& \mathrm{Yi} \mathrm{Xie}^{1}$

Finding ideal material models for studying the role of catalytic active sites remains a great challenge. Here we propose pits confined in an atomically thin sheet as a platform to evaluate carbon monoxide catalytic oxidation at various sites. The artificial three-atomic-layer thin cerium(IV) oxide sheet with approximately $20 \%$ pits occupancy possesses abundant pit-surrounding cerium sites having average coordination numbers of 4.6 as revealed by $X$-ray absorption spectroscopy. Density-functional calculations disclose that the four- and five-fold coordinated pit-surrounding cerium sites assume their respective role in carbon monoxide adsorption and oxygen activation, which lowers the activation barrier and avoids catalytic poisoning. Moreover, the presence of coordination-unsaturated cerium sites increases the carrier density and facilitates carbon monoxide diffusion along the two-dimensional conducting channels of surface pits. The atomically thin sheet with surface-confined pits exhibits lower apparent activation energy than the bulk material $\left(61.7\right.$ versus $122.9 \mathrm{~kJ} \mathrm{~mol}^{-1}$ ), leading to reduced conversion temperature and enhanced carbon monoxide catalytic ability.

\footnotetext{
${ }^{1}$ Hefei National Laboratory for Physical Sciences at Microscale, Collaborative Innovation Centre of Chemistry for Energy Materials, University of Science and Technology of China, Hefei 230026, China. ${ }^{2}$ National Synchrotron Radiation Laboratory, University of Science and Technology of China, Hefei, Anhui 230029, China. ${ }^{3}$ Division of Materials Science, Nanyang Technological University, 50 Nanyang Avenue, Singapore 639798, Singapore. ${ }^{\star}$ These authors contributed equally to this work. Correspondence and requests for materials should be addressed to Y.X. (email: yxie@ustc.edu.cn) or to S.W. (email: sqwei@ustc.edu.cn).
} 
C atalysis can accelerate the speed of chemical reactions through changing the reaction pathway and lowering the activation energy, hence its wide application in diverse fields such as industrial ammonia production in Haber process ${ }^{1}$, the catalytic converters in automobiles ${ }^{2}$ and the enzymatic catalytic processes in living systems ${ }^{3}$. According to the basic theory originally proposed by Taylor, in gas-phase catalytic systems ${ }^{4}$, catalytic reactions usually occur at the active sites of catalysts, for which the crystalline defects such as step edges and kink sites with low coordination numbers are the favourable catalytic reaction centres ${ }^{5-8}$. Heterogeneous processes, generally consist of three consecutive steps of adsorption, chemical reaction and desorption, and the corresponding catalytic rate is often limited by one elementary step, referred to the rate-determining step $^{8,9}$. Taking the extensively studied catalytic CO oxidation as an example, the catalytic reaction rate is typically limited by the adsorption and dissociation of $\mathrm{O}_{2}$ molecules on the active sites of catalysts $^{10}$.

Up to now, various reaction mechanisms for $\mathrm{CO}$ oxidation have been proposed, among which the three most popular mechanisms are the Langmuir-Hinshelwood mechanism ${ }^{8,11,12}$, the Eley-Rideal mechanism $^{8,13}$ and the Mars-van Krevelen mechanism ${ }^{14}$. Although these mechanisms have been proposed to investigate the catalytic reaction processes, atomic-level insights into the underlying mechanism of catalysis are less explored. This is mainly due to the large differences between idealized models and real catalysts, and hence the knowledge gained from these models is not readily applicable to real catalysts. To evaluate where catalysis occurs and which site has the highest activity, simplifying and bridging the catalyst model with the real catalyst is of paramount importance. Recently, for better understanding the fundamental mechanism in catalytic $\mathrm{CO}$ oxidation, reactions on oriented crystals with a predominantly exposed facet have been further explored ${ }^{15-23}$, thanks to the single active site on these facets. For instance, theoretical calculations revealed that $\mathrm{CO}$ had stronger chemisorption on the $\{110\}$ and $\{100\}$ facets of $\mathrm{CeO}_{2}$ than on the $\{111\}$ facets ${ }^{17,18}$. This has been experimentally demonstrated by the fact that the rod-like $\mathrm{CeO}_{2}$ exposing $\{110\}$ and $\{100\}$ facets showed obviously improved $\mathrm{CO}$ catalytic properties than octahedron-like $\mathrm{CeO}_{2}$ exposing $\{111\}$ facets ${ }^{19}$. Also, Xie et al. ${ }^{20}$ observed that the cube-like $\mathrm{CeO}_{2}$ crystals with exposed $\{100\}$ facets exhibited superior $\mathrm{CO}$ oxidation properties than the truncated octahedron-like $\mathrm{CeO}_{2}$ crystals with exposed $\{100\}$ and $\{111\}$ facets. Although the unique crystal plane has provided a good platform for understanding the role of active sites in catalysis, the relatively low ratio of surface active sites to the total atom numbers makes the quantification of a modelperformance relationship a great challenge (Fig. 1a). Fortunately, recent studies have revealed that nanoscale crystallites such as $\mathrm{CeO}_{2}$ nanostructures are beneficial to the $\mathrm{CO}$ oxidation reaction thanks to their downsizing effect ${ }^{24-29}$. For example, Botana et al. ${ }^{25}$ have found that $\mathrm{CeO}_{2}$ nanotubes exhibited 400 -fold higher $\mathrm{CO}$ oxidation compared to polycrystalline $\mathrm{CeO}_{2}$ powder owing to their superior redox properties. Also, Tsang et al. have demonstrated that the quantum size effect could enable the nanosize $\mathrm{CeO}_{2}$ (below $5 \mathrm{~nm}$ ) to possess dramatically increased surface superoxide species ${ }^{30}$, which undoubtedly contributed to improve the catalytic oxidation activity ${ }^{30-32}$. In addition, Cormatt et al. have illustrated that nanocrystalline $\mathrm{CeO}_{2}$ could increase the activity of $\mathrm{Au}$ for $\mathrm{CO}$ oxidation by two orders of magnitude through effectively adsorbing and activating $\mathrm{O}_{2}$ molecules ${ }^{24}$. These results indicated that nanoscale size effects could provide more active sites and hence helped to improve the catalytic properties ${ }^{33,34}$. Hence, developing a material model with exposed unique crystal plane as well as nanoscale thickness holds the key to disclosing the role of active sites in catalysis.
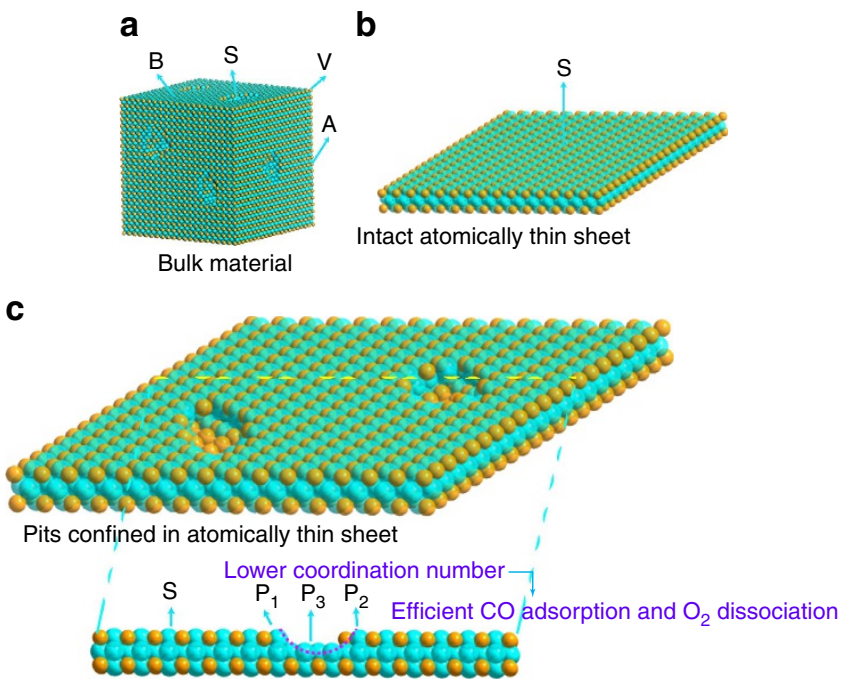

Figure 1 | Schematic illustration for evaluating catalysis location and activity. (a) Bulk $\mathrm{CeO}_{2}$; (b) intact and (c) atomically thin $\mathrm{CeO}_{2}$ sheets with surface-confined pits; $P_{1}$ and $P_{2}$ stand for the representative pitsurrounding surface $\mathrm{Ce}$ sites, while $\mathrm{P}_{3}$ represents the bottom $\mathrm{Ce}$ site of the pit; $S$ stands for the surface $C e$ site of atomically thin sheet and around the defects of bulk material; $\mathrm{A}, \mathrm{V}$ and $\mathrm{B}$ represent the arris, vertex and surface $C e$ sites of bulk material, respectively.

In this work, taking the typical $\mathrm{CO}$ oxidation catalyst of $\mathrm{CeO}_{2}$ as an example, an ideal model consisting of intact atomically thin $\mathrm{CeO}_{2}$ sheets is developed to maximize the number of coordinately unsaturated active sites, as shown in Fig. 1b. Due to the atomic thickness and specific surface area, such sheets possess the features of relatively disperse active sites and a high ratio of surface active sites to the total atom numbers. To further promote the activity of the catalytic sites, we can make lots of artificial pits with lower coordination numbers on the surface of atomically thin $\mathrm{CeO}_{2}$ sheets (Fig. 1c). To date, it remains particularly challenging to synthesize atomically thin $\mathrm{CeO}_{2}$ sheets with surface-confined pits in a controllable manner, due to the difficulty in bond-cleavage and the absence of an intrinsic driving force for the formation of coordinatively unsaturated active sites $^{7,8}$. To meet this challenge, we herein develop an 'ultrafast open space transformation' strategy and successfully synthesize three-atom-layer thin $\mathrm{CeO}_{2}$ sheets with $\sim 20 \%$ pits occupancy. $\mathrm{X}$-ray absorption fine structure spectroscopy (XAFS) and densityfunctional calculations (DFT) disclose that the pit-surrounding Ce sites with average $\mathrm{Ce}-\mathrm{O}$ coordination numbers as low as 4.6 serve as the catalytically active centres. These centres play essential roles in $\mathrm{O}_{2}$ activation, $\mathrm{CO}$ adsorption and diffusion, which lead to decreased apparent activation energy from 122.9 to $61.7 \mathrm{~kJ} \mathrm{~mol}^{-1}$ as well as obviously reduced $\mathrm{CO}$ conversion temperatures. This study provides valuable insights into the role of active centres in catalysis through a semi-quantitative modelstructure-performance relationship, holding great promise for designing catalysts with highly efficient active centres.

\section{Results}

Characterization of ultrathin $\mathrm{CeO}_{2}$ sheets with surface pits. As depicted in Fig. 2a and Supplementary Fig. S1, the successful synthesis of ultrathin $\mathrm{CeO}_{2}$ sheets took advantage of an intermediate precursor of ultrathin $\mathrm{CeCO}_{3} \mathrm{OH}$ sheets. During the formation of this precursor, the peculiar arrangement of oleate ions around the surface of $\mathrm{Ce}^{3+}$ played a vital role: $\mathrm{Ce}^{3+}$ initially interacted with oleate ions to form Ce-oleate complexes via an 

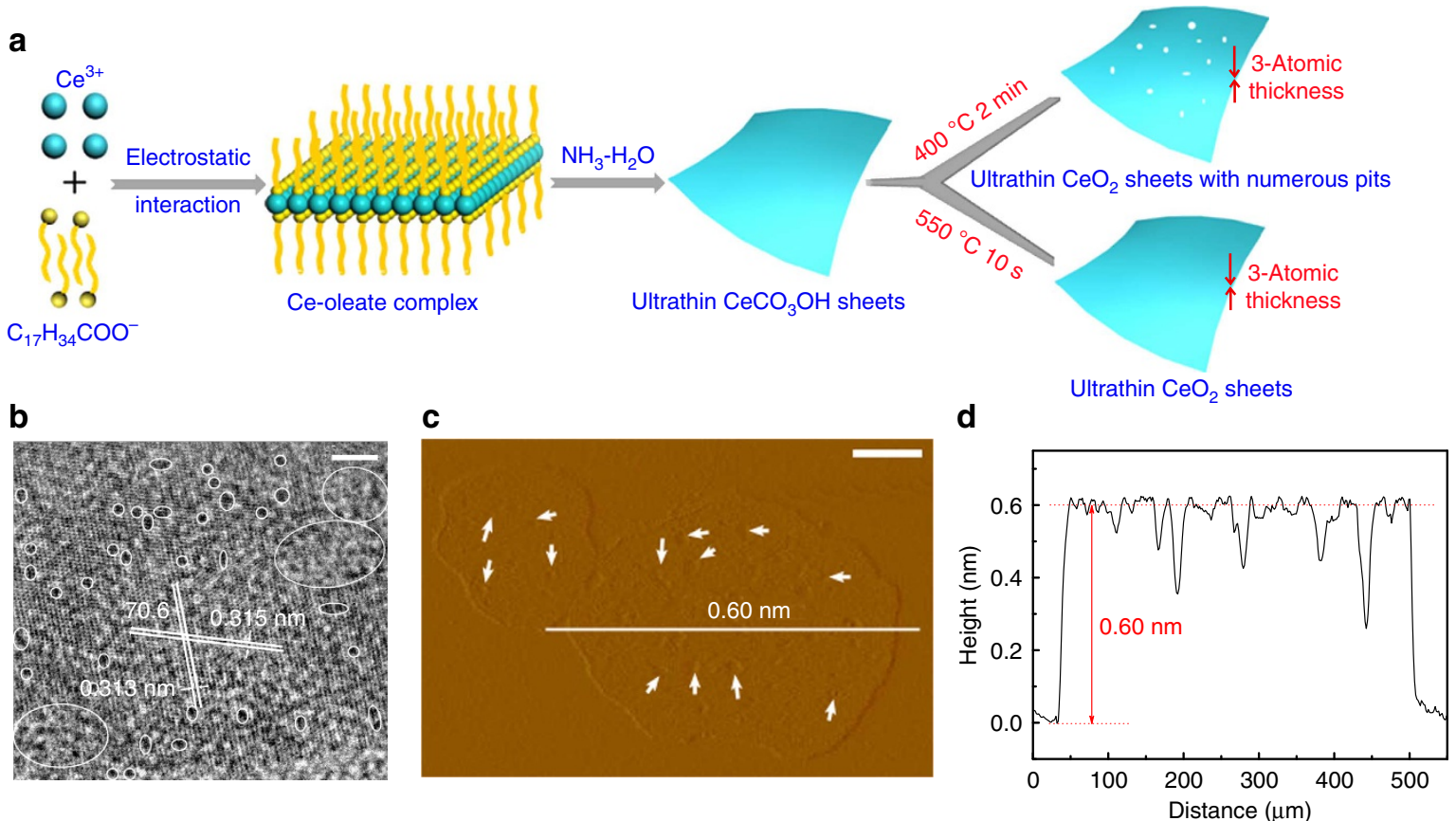

Figure 2 | Formation process and characterization of the ultrathin $\mathrm{CeO}_{\mathbf{2}}$ sheets with numerous surface pits. (a) Schematic illustration for the formation of ultrathin $\mathrm{CeO}_{2}$ sheets with numerous surface pits and intact ultrathin $\mathrm{CeO}_{2}$ sheets. Note that the heating temperature and time have a vital role in the formation of pits on their surface. (b-d) Characterizations for the three-atom-thin $\mathrm{CeO}_{2}$ sheets with numerous surface pits: (b) HRTEM image, (c) AFM image and (d) the height profile along the white line indicated in (c). The scale bars in (c) and (d) are 3 and 100 nm, respectively. The inset circles and arrows in (b) and (c) denote the presence of pits on their surface.

electrostatic interaction and hence assembled into atomically thin two-dimensional structure driven by the hydrophobic effect of oleate tails ${ }^{35}$. After addition of $\mathrm{NH}_{3} \cdot \mathrm{H}_{2} \mathrm{O}$ followed by hydrothermal treatment for $48 \mathrm{~h}$, the ultrathin $\mathrm{CeCO}_{3} \mathrm{OH}$ sheets were obtained and fully characterized in Supplementary Fig. S2. And then, direct heating of the precursor at $400{ }^{\circ} \mathrm{C}$ for $2 \mathrm{~min}$ in air not only resulted in the formation of clean three-atom-layer thin $\mathrm{CeO}_{2}$ sheets, but also brought on numerous pits on their surfaces (Fig. 2b-d and Supplementary Figs S3-S5). The high-resolution transmission electron microscopy (TEM) image in Fig. $2 \mathrm{~b}$ clearly revealed the high [110] orientation of the $\mathrm{CeO}_{2}$ sheets, while the atomic force microscopy (AFM) image and the corresponding height profile in Fig. $2 \mathrm{c}$,d showed their height of about $0.60 \mathrm{~nm}$, which fairly agreed with the $0.57 \mathrm{~nm}$ thickness of three-atom-thin $\mathrm{CeO}_{2}$ slab along the [110] direction. More importantly, the HRTEM and AFM images in Fig. $2 \mathrm{~b}-\mathrm{d}$ also depicted that the pits possessed an occupancy of about $20 \%$ in the three-atom-thick $\mathrm{CeO}_{2}$ sheets, indicating the presence of abundant pit-surrounding $\mathrm{Ce}$ sites. By contrast, the heating at higher temperature of $550^{\circ} \mathrm{C}$ with a shorter time of $10 \mathrm{~s}$ only resulted in the formation of clean three-atom-thick $\mathrm{CeO}_{2}$ sheets without surface pits (Fig. 2a and Supplementary Fig. S6), strongly suggesting that the heating temperature and time played an important role in the formation of pits on the surfaces of ultrathin $\mathrm{CeO}_{2}$ sheets (Supplementary Figs S7-S9).

Synchrotron radiation XAFS measurements. To obtain quantitative structural parameters around the pits of the three-atom-thick $\mathrm{CeO}_{2}$ sheets, XAFS measurements at $\mathrm{Ce} L_{3}$-edge were carried out at the U7C beamline of the National Synchrotron Radiation Laboratory, China. As shown in Fig. 3a, the Ce $L_{3}$-edge $k^{2} \chi(k)$ oscillation curve for the ultrathin $\mathrm{CeO}_{2}$ sheets with numerous surface pits displayed obvious differences compared with the ultrathin $\mathrm{CeO}_{2}$ sheets and bulk $\mathrm{CeO}_{2}$ (Supplementary Fig. S10). This was further verified by their corresponding Fourier transformed (FT) $k^{2} \chi(k)$ functions in Fig. $3 b$, which displayed that the two main peaks gradually shifted to the low $\mathrm{R}$ direction along with obviously decreased intensities, qualitatively revealing their distinct local atomic arrangement. Moreover, to achieve quantitative structural parameters around $\mathrm{Ce}$ atoms, a least-squares curve fitting was conducted and the details of the EXAFS data fitting are provided in the Methods section. As for the ultrathin $\mathrm{CeO}_{2}$ sheets, Table 1 revealed that the distances and coordination numbers for $\mathrm{Ce}-\mathrm{O}, \mathrm{Ce}-\mathrm{Ce}, \mathrm{Ce}-\mathrm{O}-\mathrm{O}$ and Ce-Ce-O coordinations reduced remarkably, while their disorder degrees increased significantly as compared with bulk counterpart, suggesting a noticeable distortion on their surface. Importantly, the pit-surrounding $\mathrm{Ce}-\mathrm{O}$ distances, confined in the ultrathin $\mathrm{CeO}_{2}$ sheets with numerous surface pits, were contracted from 2.327 to $2.302 \AA$, and their coordination numbers remarkably decreased from 6.5 to 4.6 , associated with significantly increased disorder degrees relative to the ultrathin $\mathrm{CeO}_{2}$ sheets (Table 1). This implied an obvious distortion surrounding the pits of ultrathin $\mathrm{CeO}_{2}$ sheets. Notably, such surface distortion has already been observed in previously reported atomically thin twodimensional sheets and has also been demonstrated to endow them with excellent structural stability and increased states density ${ }^{6,7,36}$. As depicted by the calculated densities of states in Fig. 3c, the presence of abundant coordination-unsaturated pitsurrounding $\mathrm{Ce}$ sites in the ultrathin $\mathrm{CeO}_{2}$ sheets led to the formation of defect states near the Fermi level, which resulted in an obvious increase of hole carrier concentration with respect to the ultrathin $\mathrm{CeO}_{2}$ sheets and bulk counterpart ${ }^{37}$. As will be shown, these features enabled the ultrathin $\mathrm{CeO}_{2}$ sheets with numerous surface pits to realize fast $\mathrm{CO}$ diffusion along the twodimensional conducting channel of surface pits and hence improved their CO catalytic properties ${ }^{6,7,38}$. 


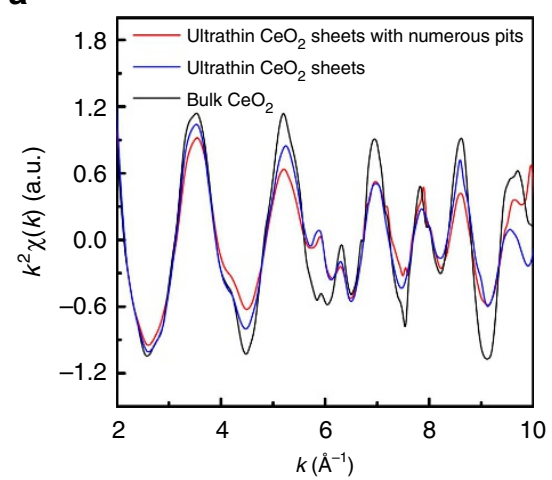

b

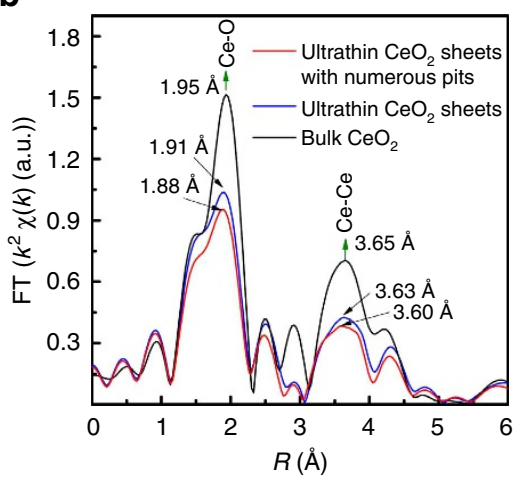

C

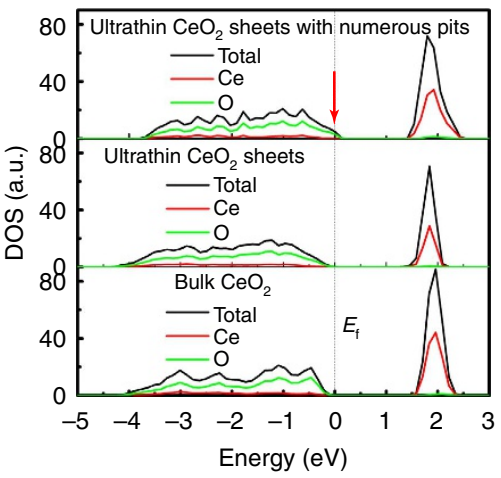

Figure 3 | Synchrotron radiation XAFS measurements. (a) $C e L_{3}$-edge extended XAFS oscillation function $k^{2} \chi(k)$, (b) the corresponding Fourier transforms $\mathrm{FT}\left(k^{2} \chi(k)\right)$, (c) the calculated densities of states for the ultrathin $\mathrm{CeO}_{2}$ sheets with numerous surface pits, ultrathin $\mathrm{CeO}$ sheets and bulk $\mathrm{CeO}_{2}$, respectively. a.u., arbitrary unit.

Table 1 | EXAFS curve-fitting results.

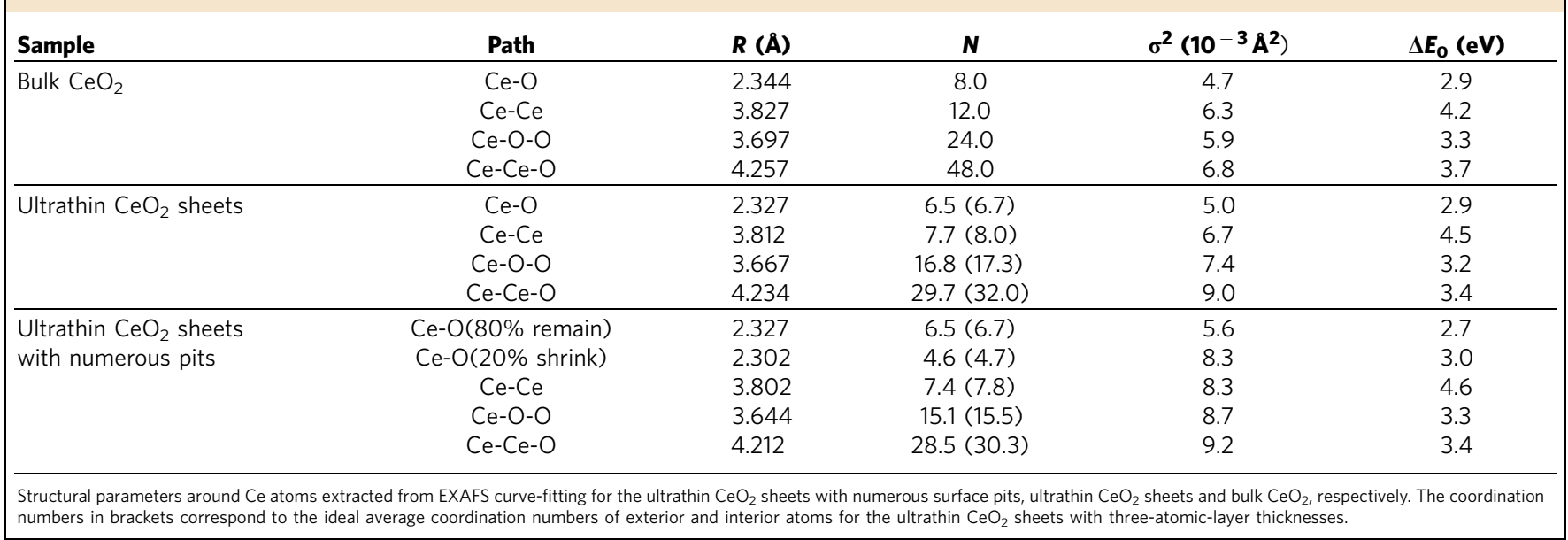

Catalytic carbon monoxide oxidation. Summarizing the above XAFS, TEM and AFM results, an ideal model for three-atomthick $\mathrm{CeO}_{2}$ sheets with surface-confined pits is built to gain atomic-level insights into the underlying catalytic mechanism (Fig. 4c). According to the widely accepted Langmuir-Hinshelwood mechanism $8,11,12$, the $\mathrm{CO}$ oxidation occurring on the pitsurrounding $\mathrm{Ce}$ sites may consist of the following elementary steps: (1) Adsorption of $\mathrm{CO}$ and $\mathrm{O}_{2}$ molecules from the gas phase onto the pit-surrounding $\mathrm{Ce}$ sites; (2) dissociation of $\mathrm{O}_{2}$ molecules on the pit-surrounding Ce sites; (3) reaction between $\mathrm{CO}$ and dissociated $\mathrm{O}$ atom to form $\mathrm{CO}_{2}$; (4) desorption of $\mathrm{CO}_{2}$ to the gas phase. In addition, it is noticeable that $\mathrm{CO}$ should diffuse to the nearby atomic $\mathrm{O}$ before their reaction to form $\mathrm{CO}_{2}$, in which the $\mathrm{CO}$ molecules need to overcome a diffusion barrier ${ }^{39,40}$. DFT calculations were performed on various pitsurrounding Ce sites and on-surface Ce sites in order to identify the active sites. Figure $4 \mathrm{a}$ clearly revealed that $\mathrm{CO}$ and $\mathrm{O}_{2}$ molecules exhibited the highest adsorptive energies (absolute values) at pit-surrounding 4 -coordinated Ce site $\left(\mathrm{P}_{2},-1.25 \mathrm{eV}\right)$ and 5-coordinated $\mathrm{Ce}$ site $\left(\mathrm{P}_{1},-1.75 \mathrm{eV}\right)$, respectively. This indicated that the $\mathrm{CO}$ and $\mathrm{O}_{2}$ molecules preferred to adsorb at the pit-surrounding Ce sites with different coordination numbers and hence would not block the active sites for each other, thus effectively avoiding the catalyst poisoning. Also, the dissociation of $\mathrm{O}_{2}$ molecules needed the lowest activation energy of $0.46 \mathrm{eV}$ at the two adjacent 5 -coordinated Ce sites $\left(2 \mathrm{P}_{1}\right.$, Fig. $\left.4 \mathrm{~b}\right)$, suggesting that they preferably dissociated into oxygen atoms on the dual adjacent fivefold coordinated $\mathrm{Ce}$ sites and their corresponding transition state was shown in Fig. 4c. Considering the relatively small energy barrier of $0.12 \mathrm{eV}$ for the $\mathrm{CO}+\mathrm{O}$ associative desorption, it was suggested that the bonding strength for $\mathrm{O}$ atoms at the pit-surrounding $\mathrm{Ce}$ sites was optimum and would make these sites active ${ }^{5}$. The above results revealed that the coordination number and spatial distribution of the pitsurrounding $\mathrm{Ce}$ sites played a crucial role in $\mathrm{CO}$ catalytic oxidation. In this case, the pits with different size and shape would theoretically achieve the similar $\mathrm{CO}$ adsorption energies and $\mathrm{O}_{2}$ activation energies when their pits could provide the 4- and 5-coordinated pit-surrounding $\mathrm{Ce}$ sites with similar percentage and spatial distribution (Supplementary Fig. S11). Moreover, the $\mathrm{CO}$ diffusion barriers on the pit-surrounding $\mathrm{Ce}$ sites and on-surface Ce sites were calculated to be 0.08 and $0.17 \mathrm{eV}$, respectively. The lowered $\mathrm{CO}$ diffusion barrier on the pitsurrounding Ce sites could be benefited from their increased states density near the Fermi level (Fig. 3c) and hence contributed to lower the overall activation energy, thus finally improving their $\mathrm{CO}$ oxidation activity. In this case, the adsorbed $\mathrm{CO}$ molecules at 4-coordinated $\mathrm{Ce}$ site could effectively diffuse into the 5-coordinated $\mathrm{Ce}$ site along the two-dimensional conducting channel of surface pits and hence reacted with the dissociated oxygen atoms to produce $\mathrm{CO}_{2}$. Thus, although it was difficult to distinguish the rate-determining step and accurately evaluate the 
a

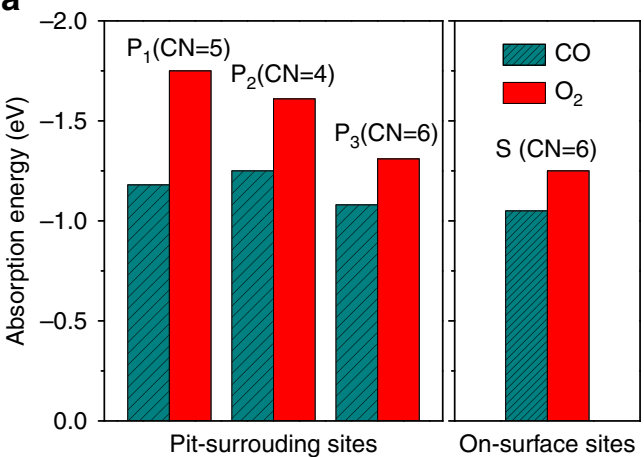

b

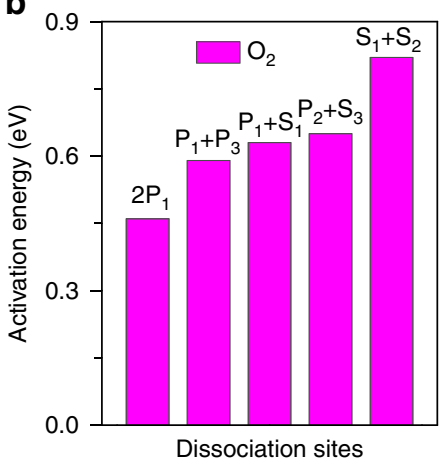

C
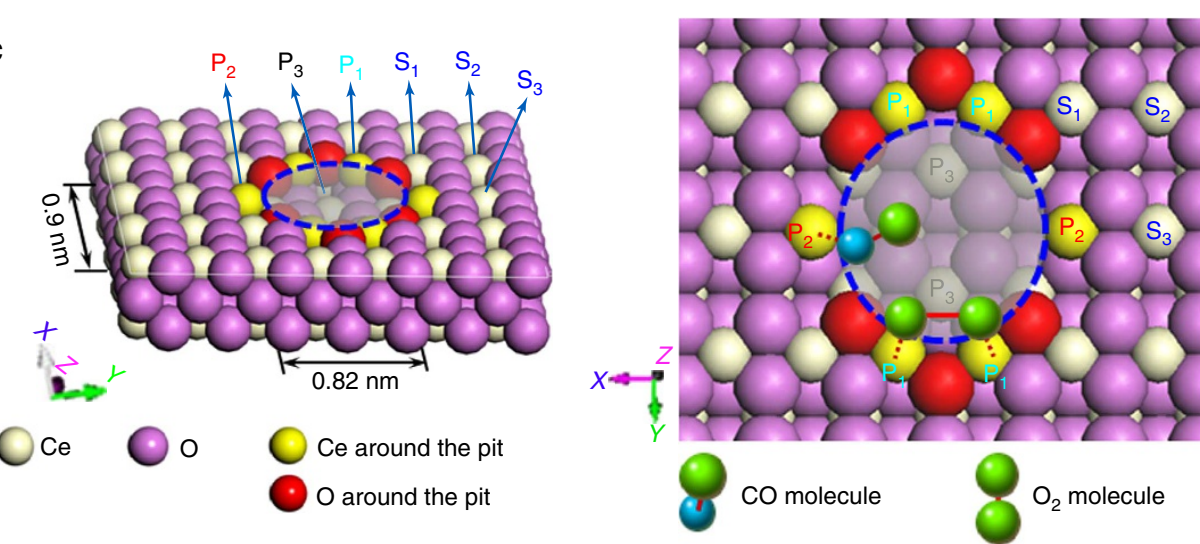

d

e
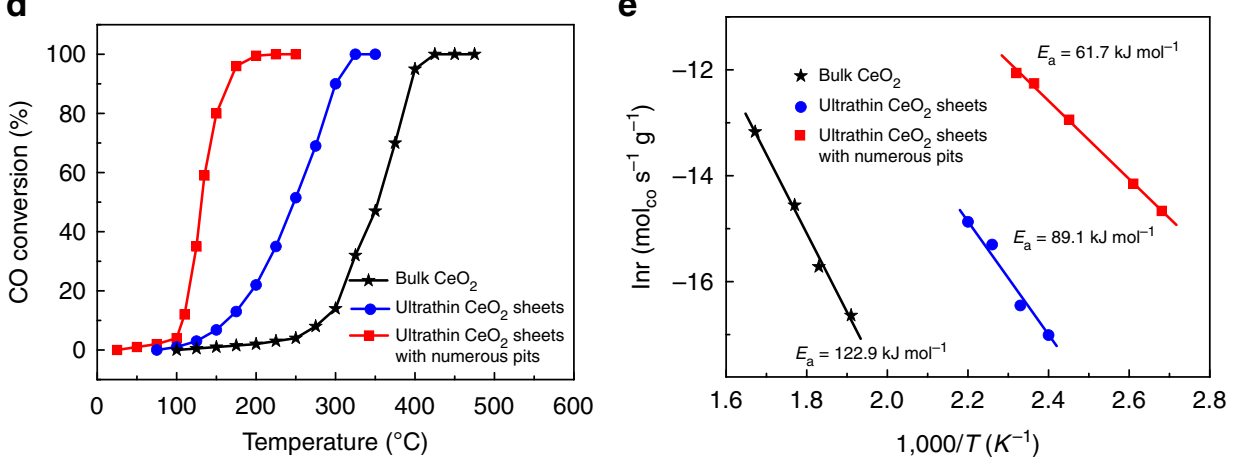

Figure 4 | Catalytic carbon monoxide oxidation. (a) Calculated adsorption energies for $\mathrm{CO}$ and $\mathrm{O}_{2}$ molecules on the pit-surrounding Ce sites $\left(\mathrm{P}_{1} \sim \mathrm{P}_{3}\right)$ and on-surface $\mathrm{Ce}$ sites $\left(\mathrm{S}_{1} \sim \mathrm{S}_{3}\right)$, and $(\mathbf{b})$ calculated activation energies for $\mathrm{O}_{2}$ dissociation on the adjacent $\mathrm{Ce}$ sites of ultrathin $\mathrm{CeO} \mathrm{O}_{2}$ sheets with numerous pits; the coordination numbers of $P_{1}, P_{2}, P_{3}, S_{1}, S_{2}$ and $S_{3}$ are $5,4,6,6,6$ and 6 , respectively. (c) Overhead and top views of schematic structures for the three-atom-thick $\mathrm{CeO}_{2}$ sheets with a pit size of $0.90 \mathrm{~nm} \times 0.82 \mathrm{~nm}$ (This size is fairly consistent with that statistically calculated from the HRTEM image in Fig. 2b); the $\mathrm{CO}$ molecules prefer to adsorb at $\mathrm{P}_{2}$ site, while the $\mathrm{O}_{2}$ molecules tend to adsorb and dissociate at the adjacent $\mathrm{P}_{1}$ sites. (d) Catalytic activity for $\mathrm{CO}$ oxidation versus reaction temperatures (experimental error: $\pm 3 \%$ ) and (e) the corresponding Arrhenius plot for the three $\mathrm{CeO}_{2}$ samples (experimental error: $\pm 3 \%$ ).

overall activation energy without full kinetic simulations, the simple analysis of the above activation energies helped to draw the following conclusions: (1) The step of $\mathrm{O}_{2}$ activation was the rate-limiting step for the ultrathin $\mathrm{CeO}_{2}$ sheets with numerous surface pits, since the calculated $\mathrm{O}_{2}$ activation energy of $\sim 0.46 \mathrm{eV}$ on the pit-surrounding $\mathrm{Ce}$ sites was much higher than the corresponding calculated $\mathrm{CO}+\mathrm{O}$ associative desorption energy $(0.12 \mathrm{eV})$ and the CO diffusion energy $(0.08 \mathrm{eV}) ;(2)$ the overall activation energy on the pit-surrounding $\mathrm{Ce}$ sites could be roughly evaluated to be the value between 0.46 and $0.66 \mathrm{eV}$ (corresponding to the range from 44.4 to $63.6 \mathrm{~kJ} \mathrm{~mol}^{-1}$ ), fairly consistent with their apparent activation energy of $61.7 \mathrm{~kJ} \mathrm{~mol}^{-1}$ in Fig. 4e. Based on the above results, one could conclude that the ultrathin $\mathrm{CeO}_{2}$ sheets with numerous surface pits had $\sim 20 \%$ pits occupancy, in which the abundant pit-surrounding Ce sites served as the active sites in $\mathrm{CO}$ catalytic oxidation and such a large amount of highly active pit-surrounding Ce sites could in fact support them to quickly complete the $\mathrm{CO}$ catalytic oxidation at a low reaction temperature (Fig. 4d). By contrast, for the intact ultrathin $\mathrm{CeO}_{2}$ sheets without surface pits and bulk counterpart (Supplementary Figs S6 and S10), only the surface Ce atoms could serve as the active sites to catalyse $\mathrm{CO}$ oxidation. Note that the pit-surrounding Ce site has an average coordination number of 4.6, much smaller than that (6.5) of the surface Ce site in the ultrathin $\mathrm{CeO}_{2}$ sheets (Fig. 3), reasonably exhibiting higher catalytic activity than that at the on-surface $\mathrm{Ce}$ site. The conclusion could be further confirmed by their corresponding adsorption energies for $\mathrm{CO}$ and $\mathrm{O}_{2}$ molecules as well as $\mathrm{O}_{2}$ dissociation energies in Fig 4a,b. In this case, the ultrathin $\mathrm{CeO}_{2}$ sheets with numerous surface pits could theoretically possess 
lowered overall activation energy as well as accelerated catalytic reaction rate compared with the ultrathin $\mathrm{CeO}_{2}$ sheets without surface pits and the bulk counterpart.

To verify the predictions from the above theoretical model, the corresponding $\mathrm{CO}$ catalytic properties of the ultrathin $\mathrm{CeO}_{2}$ sheets with numerous surface pits were investigated. As shown in Fig. 4 d, they possessed $50 \%$ CO conversion at $131^{\circ} \mathrm{C}$, which was $117^{\circ} \mathrm{C}$ and $223^{\circ} \mathrm{C}$ lower than that of the ultrathin $\mathrm{CeO}_{2}$ sheets and bulk counterpart, respectively. At the same temperature of $131^{\circ} \mathrm{C}$, the ultrathin $\mathrm{CeO}_{2}$ sheets and bulk $\mathrm{CeO}_{2}$ only showed $\mathrm{CO}$ conversion ratio of 3.2 and $0.89 \%$, over 15 and 50 times smaller than that of the ultrathin $\mathrm{CeO}_{2}$ sheets with numerous surface pits. Also, the complete $\mathrm{CO}$ conversion temperature for the ultrathin $\mathrm{CeO}_{2}$ sheets with numerous surface pits was about $200^{\circ} \mathrm{C}$, strikingly lower than that of the ultrathin $\mathrm{CeO}_{2}$ sheets $\left(325^{\circ} \mathrm{C}\right)$ and bulk counterpart $\left(425^{\circ} \mathrm{C}\right)$. To the best of our knowledge, the half conversion temperature for the ultrathin $\mathrm{CeO}_{2}$ sheets with numerous surface pits was much lower than all of those reported values for parent $\mathrm{CeO}_{2}$ (refs 41-44), strongly suggesting the remarkably improved low-temperature $\mathrm{CO}$ catalytic activity. Moreover, the Arrhenius plot in Fig. 4e revealed that the apparent activation energy for the ultrathin $\mathrm{CeO}_{2}$ sheets with numerous surface pits was $61.7 \mathrm{~kJ} \mathrm{~mol}^{-1}$, obviously smaller than the values of 89.1 and $122.9 \mathrm{~kJ} \mathrm{~mol}^{-1}$ for the ultrathin $\mathrm{CeO}_{2}$ sheets and bulk counterpart. This implied that the $\mathrm{CO}$ oxidation catalysed by the ultrathin $\mathrm{CeO}_{2}$ sheets with numerous surface pits involved different catalytically active sites in comparison with the other two $\mathrm{CeO}_{2}$ samples ${ }^{45}$, in which the 4-coordinated and 5-coordinated pit-surrounding $\mathrm{Ce}$ sites undoubtedly accounted for the eminently lowered apparent activation energy and subsequently decreased CO conversion temperatures. Accordingly, the obviously promoted $\mathrm{CO}$ catalytic performances for ultrathin $\mathrm{CeO}_{2}$ sheets with numerous surface pits were fairly consistent with the corresponding theoretical model predictions, providing strong evidence for the validity and reliability of the designed model. Of note, as shown in Supplementary Fig. S12, one can conclude that the ultrathin $\mathrm{CeO}_{2}$ sheets with surfaceconfined pits possessed two types of active sites including very active pit-surrounding $\mathrm{Ce}$ atoms and less active surface $\mathrm{Ce}$ atoms: when the surface pits had a low percentage such as $2 \%$ (Supplementary Fig. S7), the small amount of pit-surrounding $\mathrm{Ce}$ atoms and other surface $\mathrm{Ce}$ atoms could together act as the active sites to catalyse the $\mathrm{CO}$ oxidation reactions; whereas, when the pits occupancy in the ultrathin $\mathrm{CeO}_{2}$ sheets was up to $10 \%$, the pit-surrounding $\mathrm{Ce}$ atoms could serve as the preferentially active sites to support them quickly to complete the CO oxidation reaction in a narrow temperature range (Supplementary Fig. S9).

\section{Discussion}

As is well-known, the auto-ignition temperature of $\mathrm{CO}$ molecules is as high as $608.89^{\circ} \mathrm{C}$. After adding a catalyst such as bulk $\mathrm{CeO}_{2}$, the ignition temperature corresponding to $10 \% \mathrm{CO}$ conversion could be reduced to $281{ }^{\circ} \mathrm{C}$ (Fig. $4 \mathrm{~d}$ ), verifying that the catalyst could indeed increase the reaction rate for $\mathrm{CO}$ oxidation. More interestingly, our designed three-atom-thick $\mathrm{CeO}_{2}$ sheets with surface-confined pits further lowered the ignition temperature down to $106^{\circ} \mathrm{C}$. This raises important questions: what causes the strikingly different catalytic activity in the same catalyst material? How does it function? It is noticeable that the catalysis usually occurs at the active sites and the different active sites often lead to distinct CO catalytic reactivities ${ }^{15,30,46,47}$. Here, DFT calculations in Fig. $4 a, b$ revealed that the pit-surrounding Ce sites confined in atomically thin sheets were identified as the most favourable active centres for CO catalytic oxidation. In addition, the XAFS results in Table 1 showed that the active centres possessed the average coordination numbers of 4.6 , which was much lower than that of other various active sites. Thus, it was the peculiar active centres with lower coordination numbers that brought about the enhanced $\mathrm{CO}$ oxidation. In the active centres, the 4- and 5-coordinated pit-surrounding $\mathrm{Ce}$ sites favoured for $\mathrm{CO}$ adsorption and $\mathrm{O}_{2}$ activation, respectively, which effectively decreased the catalyst poisoning chance. Also, the highly coordination-unsaturated pit-surrounding $\mathrm{Ce}$ sites resulted in the increased carrier density near the Fermi level (Fig. 3c), which ensured fast $\mathrm{CO}$ diffusion along the two-dimensional conducting channel of surface pits and subsequently facilitated $\mathrm{CO}$ oxidation by the dissociated $\mathrm{O}$ atoms ${ }^{6,7,32,48}$. In other words, the active centres consisting of pit-surrounding $\mathrm{Ce}$ sites were beneficial to $\mathrm{O}_{2}$ activation, $\mathrm{CO}$ adsorption and diffusion. In addition, the extremely high fraction of surface Ce atoms as well as the quantum size effect in the ultrathin $\mathrm{CeO}_{2}$ sheets could also contribute to lower their overall activation energy and definitely accelerate the speed of CO catalytic reaction compared with the bulk counterpart ${ }^{24-32}$, which could be verified by the $\mathrm{CO}$ conversion curves and the corresponding activation energies of the intact ultrathin $\mathrm{CeO}_{2}$ sheets and bulk counterpart in Fig. 4d,e. As such, the different activation energies between the ultrathin $\mathrm{CeO}_{2}$ sheets with numerous surface pits and the bulk counterpart were not only due to the presence of surface pits but also due to the quantum effects at the nano-size. That is to say, in the ultrathin $\mathrm{CeO}_{2}$ sheets with numerous surface pits, the quantum size effect and the abundant highly active pit-surrounding Ce sites could contribute to lower their overall activation energy and definitely increase the speed of $\mathrm{CO}$ catalytic reaction. Actually, the experimental results in Fig. 4d,e showed that the apparent activation energy for the ultrathin $\mathrm{CeO}_{2}$ sheets with numerous surface pits remarkably decreased from 122.9 to $61.7 \mathrm{~kJ} \mathrm{~mol}^{-1}$, and the corresponding $\mathrm{CO}$ conversion temperatures also reduced dramatically as compared to the bulk counterpart, verifying the predictions of the theoretical model. As a consequence, we bridged the gap between theoretical model and real catalyst, and subsequently identified the catalytically active centre through semi-quantifying the model-structure-performance relationship, which deepened our understanding about the underlying nature of catalysis.

In conclusion, conceptually new atomically thin sheets with surface-confined pits were proposed as an excellent platform to study the role of active sites in catalysis. Freestanding three-atomthick $\mathrm{CeO}_{2}$ sheets with $\sim 20 \%$ surface pits occupancy were synthesized via an 'ultrafast open space transformation' strategy and taken as an example to semi-quantify the model-structureperformance relationship. XAFS results directly evidenced the lower average coordination number of 4.6 for the abundant pitsurrounding Ce sites, which in fact served as the catalytically active centres. DFT calculations demonstrated that the 4 - and 5 -coordinated pit-surrounding Ce sites performed their respective functions in $\mathrm{CO}$ adsorption and $\mathrm{O}_{2}$ activation, thus reducing the activation barrier and decreasing the catalyst poisoning chance. Also, the highly coordination-unsaturated pit-surrounding $\mathrm{Ce}$ sites brought about the increased hole carrier density and hence assured fast $\mathrm{CO}$ diffusion along the two-dimensional conducting channel of surface pits. As a result, the atomically thin $\mathrm{CeO}_{2}$ sheets with surface-confined pits lowered the apparent activation energy from 122.9 to $61.7 \mathrm{~kJ} \mathrm{~mol}^{-1}$ and definitely decreased the $\mathrm{CO}$ conversion temperatures. This work provides crucial insights into the role of active centres in catalysis through first semiquantifying the model-structure-performance relationship, opening the door for designing catalytic centres.

\section{Methods}

Synthesis details. Synthesis of freestanding ultrathin $\mathrm{CeCO}_{3} \mathrm{OH}$ sheets: $1.5 \mathrm{mmol}$ sodium oleate was initially dissolved in $20 \mathrm{ml}$ distilled water. Then, $1 \mathrm{mmol}$ 
$\mathrm{CeCl}_{3} \cdot 6 \mathrm{H}_{2} \mathrm{O}$ was dissolved in $10 \mathrm{ml}$ distilled water and injected to the above solution followed by vigorous stirring for $30 \mathrm{~min}$. After adding $9 \mathrm{ml} \mathrm{NH_{3 }} \cdot \mathrm{H}_{2} \mathrm{O}$ dropwise to the above solution and stirring for $1 \mathrm{~h}$, the mixture was transferred into a $50 \mathrm{ml}$ Teflon-lined autoclave, sealed and heated at $180^{\circ} \mathrm{C}$ for $48 \mathrm{~h}$. The system was then allowed to cool down to room temperature naturally, the final product was collected by centrifuging the mixture, washed with cyclohexane for many times and then dried in vacuum overnight for further characterization.

Synthesis of freestanding ultrathin $\mathrm{CeO}_{2}$ sheets with numerous surface pits: the as-obtained white ultrathin $\mathrm{CeCO}_{3} \mathrm{OH}$ sheets were directly heated at $400{ }^{\circ} \mathrm{C}$ for $2 \mathrm{~min}$ in air and then cooled to room temperature. The brilliant yellow powders were collected for further characterization.

Synthesis of freestanding intact ultrathin $\mathrm{CeO}_{2}$ sheets: the as-obtained white ultrathin $\mathrm{CeCO}_{3} \mathrm{OH}$ sheets were directly heated at $550{ }^{\circ} \mathrm{C}$ for $10 \mathrm{~s}$ in air and then cooled to room temperature. The brilliant yellow powders were collected for further characterization.

Characterization. XRD patterns were recorded by using a Philips X'Pert Pro Super diffractometer with $\mathrm{Cu} K \alpha$ radiation $(\lambda=1.54178 \AA)$. TEM images and highresolution TEM image were performed by using a JEOL-2010 TEM with an acceleration voltage of $200 \mathrm{kV}$. The field emission scanning electron microscopy images were performed by using a FEI Sirion-200 SEM. AFM study in the present work was performed by means of Veeco DI Nano-scope MultiMode V system. X-ray photoelectron spectra (XPS) were acquired on an ESCALAB MKII with $\mathrm{Mg}$ $\mathrm{K} \alpha(\mathrm{h} v=1253.6 \mathrm{eV})$ as the excitation source. The binding energies obtained in the XPS spectral analysis were corrected for specimen charging by referencing $\mathrm{C} 1 \mathrm{~s}$ to $284.5 \mathrm{eV}$. The IR spectra were measured on a NICOLET FT-IR spectrometer, using pressed $\mathrm{KBr}$ tablets. Raman spectra were detected by a RenishawRM3000 MicroRaman system. Thermal gravimetric analysis of the as-synthesized samples was carried out on a Shimadzu TA-50 thermal analyser at a heating rate of $10^{\circ} \mathrm{C}$ $\mathrm{min}^{-1}$ from room temperature to $800^{\circ} \mathrm{C}$. The Ce $L_{3}$-edge XAFS was measured at the 1W1B beamline of Beijing Synchrotron Radiation Factory (BSRF) and the U7C beamline of the National Synchrotron Radiation Laboratory, China. The storage ring of the BSRF was operated at $2.5 \mathrm{GeV}$ with a maximum current of $250 \mathrm{~mA}$, and National Synchrotron Radiation Laboratory was operated with a storage ring working at the energy of $800 \mathrm{MeV}$ and a maximum electron current of $300 \mathrm{~mA}$

EXAFS data fitting. Note that the HRTEM result in Fig. $2 b$ clearly reveals that the pits possess an occupancy of about $20 \%$ in the ultrathin $\mathrm{CeO}_{2}$ sheets with numerous surface pits. As such, $20 \% \mathrm{Ce}-\mathrm{O}$ distances and coordination numbers surrounding the pits of the ultrathin $\mathrm{CeO}_{2}$ sheets are regarded to be shrunk during their EXAFS curve-fitting, while the other $80 \% \mathrm{Ce}-\mathrm{O}$ distances and coordination numbers still remain the same as that of intact ultrathin $\mathrm{CeO}_{2}$ sheets without surface pits.

As shown in Fig. 3b, the FT curve of bulk $\mathrm{CeO}_{2}$ exhibited two main peaks at 1.95 and $3.65 \AA$, corresponding to the nearest $\mathrm{Ce}-\mathrm{O}$ and next nearest $\mathrm{Ce}-\mathrm{Ce}$ coordinations, respectively. For the ultrathin $\mathrm{CeO}_{2}$ sheets, the $\mathrm{Ce}-\mathrm{O}$ and $\mathrm{Ce}-\mathrm{Ce}$ peak position exhibited a noticeable shift to 1.91 and $3.63 \AA$, associated with obvious reduction in their peak intensities. Contrastingly, for the ultrathin $\mathrm{CeO}_{2}$ sheets with numerous surface pits, the intensities of these two peaks further decreased significantly, and most importantly, the $\mathrm{Ce}-\mathrm{O}$ and Ce-Ce peaks were further shifted to 1.88 and $3.60 \AA$, qualitatively revealing their different local atomic arrangement compared with ultrathin $\mathrm{CeO}_{2}$ sheets and bulk counterpart. Moreover, to achieve quantitative structural parameters around $\mathrm{Ce}$ atoms in the ultrathin $\mathrm{CeO}_{2}$ sheets with numerous surface pits, a least-squares curve parameter fitting for the $\mathrm{Ce}-\mathrm{O}, \mathrm{Ce}-\mathrm{Ce}, \mathrm{Ce}-\mathrm{O}-\mathrm{O}$ and $\mathrm{Ce}-\mathrm{Ce}-\mathrm{O}$ peaks was conducted. The fitting results for the ultrathin $\mathrm{CeO}_{2}$ sheets in Table 1 revealed that the distances and coordination numbers for the $\mathrm{Ce}-\mathrm{O}, \mathrm{Ce}-\mathrm{Ce}, \mathrm{Ce}-\mathrm{O}-\mathrm{O}$ and $\mathrm{Ce}-\mathrm{Ce}-\mathrm{O}$ coordinations reduced remarkably, while their disorder degrees increased significantly compared with bulk counterpart, suggesting a noticeable distortion on their surface. By contrast, the ultrathin $\mathrm{CeO}_{2}$ sheets with numerous surface pits possessed further contracted distances and decreased coordination numbers for the $\mathrm{Ce}-\mathrm{O}, \mathrm{Ce}-\mathrm{Ce}, \mathrm{Ce}-$ $\mathrm{O}-\mathrm{O}$ and $\mathrm{Ce}-\mathrm{Ce}-\mathrm{O}$ coordinations, along with further increased disorder degrees. Especially, the $\mathrm{Ce}-\mathrm{O}$ distances surrounding the pits of the ultrathin $\mathrm{CeO}_{2}$ sheets were obviously contracted from 2.327 to $2.302 \AA$, and their coordination numbers remarkably decreased from 6.5 to 4.6 , associated with significantly increased disorder degrees compared to the ultrathin $\mathrm{CeO}_{2}$ sheets.

Calculation details. Our first-principles density functional theory (DFT) calculations were performed using a plane wave basis set with the projector augmented plane-wave method ${ }^{46-49}$. The exchange-correlation interaction is described within the generalized gradient approximation ${ }^{50,51}$. The energy cutoff is set to $400 \mathrm{eV}$, and the atomic positions are allowed to relax until the energy and force are less than $10^{-4} \mathrm{eV}$ and $5 \times 10^{-3} \mathrm{eV}^{-1}$, respectively. The ultrathin $\mathrm{CeO}_{2}$ sheets with numerous surface pits were simulated by periodically repeating the $\mathrm{CeO}$ layers along the [110] direction of the unit cell. Each sheet model consists of three $\mathrm{CeO}$ planes and is separated by a vacuum region of $15 \AA$. An elliptical void area by removing partial surface $\mathrm{Ce}$ and $\mathrm{O}$ atoms on the ultrathin sheet was used to simulate the pit. For the ultrathin $\mathrm{CeO}_{2}$ sheets, they were simulated by periodically repeating the $\mathrm{CeO}$ layers along the [110] direction of the unit cell. Each sheet model consists of three $\mathrm{CeO}$ planes and is separated by a vacuum region of $15 \AA$ The comparison calculations for bulk $\mathrm{CeO}_{2}$ were performed within supercells constructed from a standard unit cell of $\mathrm{CeO}_{2}$ lattice.

Moreover, we calculated the adsorption energy of $\mathrm{CO}$ and $\mathrm{O}_{2}$ molecules surrounding the pits for various-sized pit models. The adsorption energy $\Delta E_{\text {ads }}$ is defined as follows:

$$
\Delta E_{\text {ads }}=E_{\text {tot }}(\mathrm{NS}+\mathrm{AD})-E_{\text {tot }}(\mathrm{NS})-E_{\text {tot }}(\mathrm{AD})
$$

where $E_{\mathrm{tot}}(\mathrm{NS}+\mathrm{AD}), E_{\mathrm{tot}}(\mathrm{NS})$ and $E_{\mathrm{tot}}(\mathrm{AD})$ are the total energies of $\mathrm{CO}$ and/or $\mathrm{O}_{2}$ molecule adsorbing on the ultrathin $\mathrm{CeO}_{2}$ sheet with numerous surface pits, the ultrathin $\mathrm{CeO}_{2}$ sheet with numerous surface pits, and the adsorbent $\mathrm{CO} / \mathrm{O}_{2}$ molecules, respectively. The negative value of $\Delta E_{\text {ads }}$ implies that adsorption is exothermic, so more the negative value of the $\Delta E_{\text {ads }}$, more is the stability of the model. Furthermore, the activation barriers of $\mathrm{O}_{2}$ dissociation were calculated by the climbing image nudged elastic band method ${ }^{52,53}$, in which the zero energy reference is $\mathrm{O}_{2}$ in gas phase.

Catalytic activity measurements. The catalytic properties of $\mathrm{CO}$ oxidation for $\mathrm{CeO}_{2}$ were conducted in a quartz-tube plug flow reactor using $50 \mathrm{mg}$ catalyst in a mixed gas of $1 \mathrm{vol} \%$ CO and $99 \mathrm{vol} \%$ dried air at a flow rate of $40 \mathrm{ml} \mathrm{min}^{-1}$ corresponding to a gas hourly space velocity of $48,000 \mathrm{ml} \mathrm{h}^{-1} \mathrm{~g}^{-1}$. The catalyst was heated to the desired temperatures at a rate of $2^{\circ} \mathrm{C} \mathrm{min}^{-1}$ and then kept for $30 \mathrm{~min}$ until the catalytic reaction reached a steady state. Then, the composition of effluent gas was analysed by a portable intelligent professional $\mathrm{CO}_{2}$ detector (Keernuo Gt901, Shenzhen Keernuo Technology Co. Ltd). The CO conversion was calculated from the change in $\mathrm{CO}_{2}$ concentration of the inlet and outlet gases. Each experiment for the $\mathrm{CO}$ conversion was repeated at least three times to ensure reproducibility and to assess the range of experimental errors associated with the experiments, and the experimental error was assessed to be within $\pm 3.0 \%$.

\section{References}

1. Appl, M. 'Ammonia'. in Ullmann's Encyclopedia of Industrial Chemistry (Wiley-VCH, Weinheim, Germany, 2006).

2. Joo, S. H. et al. Size effect of ruthenium nanoparticles in catalytic carbon monoxide oxidation. Nano Lett. 10, 2709-2713 (2010).

3. Neitzel, J. J. Enzyme catalysis: the serine proteases. Nat. Edu. 3, 21 (2010).

4. Taylor, H. S. A theory of the catalytic surface. Proc. R. Soc. London, Ser. A 108 105-111 (1925)

5. Fu, Q. et al. Interface-confined ferrous centres for catalytic oxidation. Science 328, 1141-1144 (2010).

6. Sun, Y. F. et al. Freestanding tin disulfide single-layers realizing efficient visiblelight water splitting. Angew. Chem. Int. Ed. 51, 8727-8731 (2012).

7. Sun, Y. F. et al. Fabrication of flexible and freestanding zinc chalcogenide single-layers. Nat. Commun. 3, 1057 (2012).

8. Dumesic, J. A., Huber, G. W. \& Boundart, M. Principles of Heterogeneous Catalysis (Wiley-VCH, Weinheim, Germany, 2008)

9. Christopher, P., Xin, H. L. \& Linic, S. Visible-light-enhanced catalytic oxidation reactions on plasmonic silver nanostructures. Nat. Chem. 3, 467-472 (2011).

10. Deng, X. Y., Min, B. K., Guloy, A. \& Friend, C. M. Enhancement of $\mathrm{O}_{2}$ dissociation on $\mathrm{Au}(111)$ by adsorbed oxygen: implications for oxidation catalysis. J. Am. Chem. Soc. 127, 9267-9270 (2005).

11. Langmuir, $\mathrm{I}$. The mechanism of the catalytic action of platinum in the reactions $2 \mathrm{CO}+\mathrm{O}_{2}=2 \mathrm{CO}_{2}$ and $2 \mathrm{H}_{2}+\mathrm{O}_{2}=2 \mathrm{H}_{2} \mathrm{O}$. Trans Farad. Soc. 17, 621-654 (1922).

12. Hinshelwood, C. N. The Kinetics of Chemical Change (Clarendon press, Oxford, UK, 1940).

13. Eley, D. D. \& Rideal, E. K. Parahydrogen conversion on tungsten. Nature 146, 401-402 (1946)

14. Mars, P. \& van Krevelen, D. W. Oxidations carried out by means of vanadium oxide catalysts. Spec. Suppl. Chem. Eng. Sci. 3, 41-59 (1954).

15. Xie, X. W., Li, Y., Liu, Z. Q., Haruta, M. \& Shen, W. J. Low-temperature oxidation of $\mathrm{CO}$ catalysed by $\mathrm{Co}_{3} \mathrm{O}_{4}$ nanorods. Nature 458, 746-749 (2009).

16. Leng, M. et al. Polyhedral 50 -facet $\mathrm{Cu}_{2} \mathrm{O}$ microcrystals partially enclosed by $\{311\}$ high-index planes: synthesis and enhanced catalytic $\mathrm{CO}$ oxidation activity. J. Am. Chem. Soc. 132, 17084-17087 (2010).

17. Nolan, M. \& Watson, G. W. The surface dependence of CO adsorption on Ceria. J. Phys. Chem. B 110, 16600-16606 (2006).

18. Huang, M. \& Fabris, S. CO adsorption and oxidation on ceria surfaces from DFT + U calculations. J. Phys. Chem. C 112, 8643-8648 (2008).

19. Wu, Z. L., Li, M. J. \& Overbury, S. H. On the structure dependence of CO oxidation over $\mathrm{CeO}_{2}$ nanocrystals with well-defined surface planes. J. Catal. 285, 61-73 (2012)

20. Wang, X., Jiang, Z. Y., Zheng, B. J., Xie, Z. X. \& Zheng, L. S. Synthesis and shape-dependent catalytic properties of $\mathrm{CeO}_{2}$ nanocubes and truncated octahedral. CrystEngComm 14, 7579-7582 (2012).

21. Zhou, K. B., Wang, X., Sun, X. M., Peng, Q. \& Li, Y. D. Enhanced catalytic activity of ceria nanorods from well-defined reactive crystal planes. J. Catal. 229, 206-212 (2005). 
22. Han, X. G., Li, L. \& Wang, C. Synthesis of monodisperse $\mathrm{CeO}_{2}$ octahedra assembled by nano-sheets with exposed $\{001\}$ facets and catalytic property. CrystEngComm 14, 1939-1941 (2012).

23. Pan, C. S., Zhang, D. S. \& Shi, L. Y. CTAB assisted hydrothermal synthesis, controlled conversion and $\mathrm{CO}$ oxidation properties of $\mathrm{CeO}_{2}$ nanoplates, nanotubes, and nanorods. J. Solid State Chem. 181, 1298-1306 (2008).

24. Carrettin, S., Concepción, P., Corma, A., López Nieto, J. M. \& Puntes, V. F. Nanocrystalline $\mathrm{CeO}_{2}$ increases the activity of $\mathrm{Au}$ for $\mathrm{CO}$ oxidation by two orders of magnitude. Angew. Chem. Int. Ed. 43, 2538-2540 (2004).

25. González-Rovira, L. et al. Single-step process to prepare $\mathrm{CeO}_{2}$ nanotubes with improved catalytic activity. Nano. Lett. 9, 1395-1400 (2009).

26. Chen, G. et al. Interface reaction route to two different kinds of $\mathrm{CeO}_{2}$ nanotubes. Inorg. Chem. 47, 723-728 (2008).

27. Guo, M. N. et al. Nano-sized $\mathrm{CeO}_{2}$ with extra-high surface area and its activity for CO oxidation. Mater. Lett. 64, 1638-1640 (2010).

28. Lu, X. W., Li, X. Z., Qian, J. C. \& Chen, Z. G. The surfactant-assisted synthesis of $\mathrm{CeO}_{2}$ nanowires and their catalytic performance for $\mathrm{CO}$ oxidation. Powder Technol. 239, 415-421 (2013).

29. Huang, X. S. et al. Morphology effects of nanoscale ceria on the activity of $\mathrm{Au} / \mathrm{CeO}_{2}$ catalysts for low-temperature $\mathrm{CO}$ oxidation. Appl. Catal. B: Environ. 90, 224-232 (2009)

30. Xu, J. H. et al. Size dependent oxygen buffering capacity of ceria nanocrystals. Chem. Commun. 46, 1887-1889 (2010).

31. Yeung, C. M. Y. et al. Engineering Pt in ceria for a maximum metal support interaction in catalysis. J. Am. Chem. Soc. 127, 18010-18011 (2005).

32. Tsang, S. C., Bulpitt, C. D. A., Mitchell, P. C. H. \& Ramirez-Cuesta, A. R. Some new insights into the sensing mechanism of palladium promoted tin (IV) oxide sensor. J. Phys. Chem. B 105, 5737-5742 (2001).

33. Yeung, C. M. Y. \& Tsang, S. C. Noble metal core ceria shell catalysts for water gas shift reaction. J. Phys. Chem. C 113, 6074-6087 (2009).

34. Mclaren, A., Valdes-Solis, T., Li, G. Q. \& Tsang, S. C. Shape and size effects of $\mathrm{ZnO}$ nanocrystals on photocatalytic activity. J. Am. Chem. Soc. 131, 12540-12541 (2009).

35. Sun, Y. F., Xie, Y., Wu, C. Z., Zhang, S. D. \& Jiang, S. S. Aqueous synthesis of mesostructured $\mathrm{BiVO}_{4}$ quantum tubes with excellent dual response to visible light and temperature. Nano Res. 9, 620-631 (2010).

36. Sun, Y. F. et al. Atomically thick bismuth selenide freestanding single layers achieving enhanced thermoelectric energy harvesting. J. Am. Chem. Soc. 134, 20294-20297 (2012).

37. Park, J. S., Ryu, B., Moon, C. Y. \& Chang, K. J. Hole gas induced by defects in $\mathrm{Ge} / \mathrm{Si}$ core-shell nanowires. AIP Conf. Proc. 1399, 303-304 (2011).

38. Madl, M. et al. High resolution photocurrent imaging by atomic force microscopy on the example of single buried InAs quantum dots. Semicond. Sci. Technol. 25, 065010 (2010).

39. Green, I. X., Tang, W. J., Neurock, M. \& Yates, Jr J. T. Spectroscopic observation of dual catalytic sites during oxidation of $\mathrm{CO}$ on a $\mathrm{Au} / \mathrm{TiO}_{2}$ catalyst. Science 333, 736-739 (2011).

40. Qiao, B. T. et al. Single-atom catalysis of $\mathrm{CO}$ oxidation using $\mathrm{Pt}_{1} / \mathrm{FeO}_{\mathrm{x}}$. Nature Chem. 3, 634-641 (2011).

41. Wang, L., Zhang, L. F., Zhong, S. L. \& Xu, A. W. Highly uniform $\mathrm{CeO}_{2}$ hierarchical microstructures: Facile synthesis and catalytic activity evaluation. Appl. Surf. Sci. 263, 769-776 (2012).

42. Bao, H. Z. et al. Crystal-plane-controlled surface restructuring and catalytic performance of oxide nanocrystals. Angew. Chem. Int. Ed. 50, 12294-12298 (2011).
43. Yoon, B. et al. Charging effects on bonding and catalysed oxidation of $\mathrm{CO}$ on $\mathrm{Au}_{8}$ clusters on MgO. Science 307, 403-407 (2005).

44. Minato, T. et al. Investigation of the electronic interaction between $\mathrm{TiO}_{2}(110)$ surfaces and Au clusters by PES and STM. Surf. Sci. 566, 1012-1017 (2004).

45. Babu, S. et al. Dopant-mediated oxygen vacancy tuning in ceria nanoparticles. Nanotechnology 20, 085713 (2009).

46. Blochl, P. E. Projector augmented-wave method. Phys. Rev. B 50, 17953-17979 (1994).

47. Kresse, G. \& Furthmuller, J. Efficient iterative schemes for ab initio total-energy calculations using a plane-wave basis set. Phys. Rev. B 54, 11169-11186 (1996).

48. Kresse, G. \& Furthmuller, J. Efficiency of ab-initio total energy calculations for metals and semiconductors using a plane-wave basis set. Comput. Mater. Sci. 6, 15-50 (1996).

49. Kohn, W. \& Sham, L. J. Self-consistent equations including exchange and correlation effects. Phys. Rev. 140, A1133-A1138 (1965).

50. Perdew, J. P. \& Wang, Y. Accurate and simple analytic representation of the electron-gas correlation energy. Phys. Rev. B 45, 13244-13249 (1992).

51. Wang, Y. \& Perdew, J. P. Correlation hole of the spin-polarized electron gas, with exact small-wave-vector and high-density scaling. Phys. Rev. B 44, 013298-013307 (1991).

52. Henkelman, G., Uberuaga, B. P. \& Jónsson, H. A climbing image nudged elastic band method for finding saddle points and minimum energy paths. J. Chem. Phys 113, 9901-9904 (2000).

53. Ritter, M., Ranke, W. \& Weiss, W. Observation of strain-relaxation-induced size effects in p-type Si/SiGe resonant-tunneling diodes. Phys. Rev. B 57, 7140-7140 (1998).

\section{Acknowledgements}

We thank Prof. Rui Si in Shanghai Synchrotron Radiation Facility, Chinese Academy of Sciences for his valuable discussion on CO catalytic oxidation. This work was financially supported by National Nature Science Foundation (21331005, 11079004, 21201157, 11135008,11321503 ) and Chinese Academy of Science (XDB01020300).

\section{Author contributions}

Y.X., S.W. and Y.F.S. conceived the idea and co-wrote the paper. Y.F.S., S.G. and F.C.L. carried out the sample synthesis, characterization and CO catalytic measurement. Z.H.S., H.C. and Y.J. Analysed the XAFS data. Q.H.L. and H.B.S. performed the first-principles calculations. All the authors discussed the results, commented on and revised the manuscript.

\section{Additional information}

Supplementary Information accompanies this paper at http://www.nature.com/ naturecommunications

Competing financial interests: The authors declare no competing financial interests.

Reprints and permissions information is available online at http://npg.nature.com/ reprintsandpermissions/

How to cite this article: Sun, Y. et al. Pits confined in ultrathin cerium(IV) oxide for studying catalytic centres in carbon monoxide oxidation. Nat. Commun. 4:2899 doi: $10.1038 /$ ncomms3899 (2013). 